\title{
Impacto de las IFRS en la calidad del reporte financiero en Latinoamérica y el Caribe
}

\author{
The impact of IFRS on financial report quality in Latin America \\ and the Caribbean \\ Juan Camilo Cardona Montoya \\ Universidad de San Buenaventura - Medellín, Colombia
}

Recibido el 20 de septiembre de 2016; aceptado el 11 de septiembre de 2017

Disponible en Internet el 29 de Mayo de 2018

\section{Resumen}

Este artículo examina el impacto generado por la aplicación de las Normas Internacionales de Información Financiera (IFRS, por sus siglas en inglés) en la calidad del reporte financiero (calidad contable) para las economías latinoamericanas y del Caribe, utilizando cinco proxies de medición relacionados con la magnitud absoluta de los ajustes por devengo discrecionales, se valora la calidad contable durante los periodos contables 2006 a 2014. Al mismo tiempo, se examina los efectos de los factores institucionales e incentivos de la gerencia sobre el uso de dichos ajustes como mecanismo de manipulación del resultado. Los resultados evidencian que durante el periodo de aplicación de IFRS, las empresas reportan menor magnitud absoluta de los ajustes por devengo discrecionales, indicando una relación inversa entre estas dos variables; dichos resultados sugieren una menor manipulación del resultado, lo que es lo mismo, una mayor calidad del reporte financiero. Se pretende contribuir a la literatura presentando los hallazgos generalizados para la región latina y del Caribe, incluyendo en el estudio, a casi la totalidad de los países de esta región durante un amplio periodo de aplicación de IFRS, además de utilizar diferentes métricas de medición para dar mayor robustez a los hallazgos.

Código JEL: M41; M42; M48

Palabras clave: Ajustes por devengo; Calidad contable; Calidad del reporte financiero; Manipulación del resultado

*Autor para correspondencia.

Correo electrónico: surrendercamilo@gmail.com (J. C. Cardona Montoya)

La revisión por pares es responsabilidad de la Universidad Nacional Autónoma de México 


\begin{abstract}
This paper examines the impact generated for the application of International Financial Reporting Standards (IFRS) on financial report quality (accounting quality) for Latin American and Caribbean economies, by using five measurement proxies related to the magnitude of absolute discretionary accruals; accounting quality is assessed during the periods 2006 to 2014. At the same time, the effects of institutional factors and management incentives on the use of such accruals are examined as a mechanism for earnings management. The results show that during the period of application of IFRS, companies report lower magnitude of absolute discretionary accruals, indicating an inverse relationship between these two variables; these results suggest less earnings management, which is the same as higher financial report quality. It is intended to contribute to the literature by presenting generalized findings for the Latin and Caribbean region, by including in this study the majority of countries of the region over a wide period of application of IFRS, in addition to using different measurement metrics to give greater robustness to the findings.
\end{abstract}

JEL Classification: M41; M42; M48

Keywords: Accruals; Accounting quality; Financial report quality; Earnings management.

\title{
Introducción
}

El uso extendido en la aplicación de los Estándares Internacionales de Información Financiera (IFRS, por sus siglas en inglés) por una gran cantidad de naciones, ha llevado a los investigadores académicos y a los reguladores a nivel nacional e internacional por comprobar la relevancia de dichos estándares para cada país adoptante, aun considerando las diferencias institucionales en cada uno de ellos, que podrían condicionar la relevancia de los mismos. Específicamente se comprueba si dichos estándares mejoran la calidad de la información contable, a través del uso de varias expresiones o proxies de medición, los cuales, a lo largo de las últimas tres décadas, se han utilizado con mayor frecuencia no solamente para dar mayor robustez a los análisis, sino también para mejorar la comparabilidad de los resultados entre los países analizados. Los estudios generalmente, centran su atención en la aplicación de las IFRS en fuertes economías y mercados de capitales, siendo el caso de Australia, Reino Unido y los países de la Europa Continental, los países con mayor número de estudios reportados, incluso se ha evaluado la relevancia de dichos estándares para economías emergentes y mercados regulados como es el caso de China; Sin embargo, a pesar que casi la totalidad de los países latinoamericanos y del Caribe que han adoptado las IFRS como su marco normativo contable y sus respectivos mercados financieros se encuentran en continuo desarrollo, aun se carece de evidencia empírica suficiente sobre la relevancia de dichos estándares bajo un análisis multipaís; es por esto que se plantea, comprobar la calidad del reporte financiero para las economías latinoamericanas y del Caribe, a través de la magnitud de los ajustes por devengo discrecionales durante los periodos de aplicación de IFRS.

El documento se organiza como sigue: en la segunda sección se presenta la revisión de la literatura respecto a los impactos de la aplicación de las IFRS en las expresiones de medición del nivel de manipulación del resultado (modelos de medición de los ajustes por devengo discrecionales), al tiempo que se define las hipótesis a contrastar; en la tercera y cuarta sección se presenta la metodología a utilizar para testar las hipótesis y se presenta la selección y distribución de la muestra, respectivamente; por último, se presenta los resultados y las conclusiones en la quinta y sexta sección respectivamente. 


\section{Revisión de la literatura y desarrollo de hipótesis}

Cronológicamente, los primeros estudios en desarrollar un conjunto de expresiones de medición de la calidad contable a través de la observancia de los datos contables, fueron propuestos por Healy (1985) y DeAngelo (1986), quienes estiman e interpretan los ajustes por devengo totales, y la variación entre los ajustes por devengo de un año a otro respectivamente, como medida de la magnitud de la discrecionalidad ejercida por la gerencia (ajustes por devengo discrecionales). Con posterioridad Friedlan (1994), propone una modificación al modelo inicialmente sugerido por DeAngelo (1986), estimando la variación de los ajustes por devengo de un año a otro teniendo en cuenta la actividad operativa en cada periodo. A principios de los 90’s, Jones (1991) propone un nuevo modelo para estimar la discrecionalidad de la gerencia a través de los ajustes por devengo totales, y separar dicha discrecionalidad de aquellos ajustes por devengo que se consideran normales (no discrecionales); este modelo es sin duda, el punto de partida para la medición y comparación del nivel de manipulación del resultado (Earnings management) entre países, siendo hasta ahora el modelo con mayor uso internacional para analizar los cambios en la calidad contable, y sobre el que autores posteriores han sugerido modificaciones y nuevas variables para dar mayor capacidad al mismo (Dechow, Richardson y Tuna, 2003; Dechow, Sloan y Sweeney, 1995; Kothari, Leone y Wasley, 2005; Larcker y Richardson, 2004).

Uno de los primeros trabajos en utilizar la metodología propuesta por Jones (1991) al analizar los cambios en la calidad contable, fue el conducido por Van Tendeloo y Vanstraelen (2005), quienes examinan los efectos de la aplicación voluntaria de las IFRS en las empresas alemanas durante los años 1999 a 2001, encontrando que la manipulación del resultado no varía respecto a aquellas compañías no adoptantes. Este mismo estudio fue realizado nuevamente por Watrin y Ullmann (2012), considerando un periodo más amplio de observación (1994 a 2005), sin embargo los resultados bajo este estudio (uso del modelo propuesto por Dechow et $a l ., 1995)$ indican una disminución de la calidad de las ganancias respecto a las compañías que aplican los GAAP alemanes.

Por su parte Liu, Yip Yuen, Yao y Chan (2014), comparan la variación en la calidad contable entre las empresas alemanas que voluntariamente aplican IFRS y US GAAP para los periodos 1999 a 2004, y sugieren que la magnitud de los ajustes por devengo discrecionales (uso del modelo de Dechow et al., 1995) no varía significativamente entre los regímenes contables analizados; seguidamente Salewski, Teuteberg y Zülch (2014), aplican de nuevo el estudio para las empresas alemanas pero en esta ocasión, analizan la calidad contable considerando la obligatoriedad en la aplicación de las IFRS (uso del modelo propuesto por Kothari et al., 2005) al incorporar observaciones entre los años 1995 y 2014; sus resultados indican la existencia significativa de un incremento en el nivel de manipulación del resultado bajo los periodos de post-adopción, el cual se ha visto disminuido con el paso del tiempo, que de acuerdo a los autores, la experiencia en la aplicación y cambio gradual a IFRS, y el incremento en la efectividad de las medidas de refuerzo para garantizar la calidad contable (Enforcement), jugaron un rol decisivo en dicha disminución.

Los estudios de la calidad contable bajo el modelo propuesto por Jones (1991) o sus posteriores modificaciones, también fueron aplicados sobre varios países europeos considerados individualmente, siendo el caso de las empresas cotizadas en Rumania, sobre el que autores como Istrate et al. (2015) y Brad, Dobre y Ciobanu (2014), han analizado la aplicación de 
las IFRS de forma obligatoria en los estados financieros consolidados (entre 2006 y 2014) e individuales (entre 2010 a 2012) respetivamente, obteniendo evidencia que les sugiere sobre una disminución en el uso de los ajustes por devengo discrecionales para el primer caso (uso del modelo de Jones, 1991), mientras que para las cuentas anuales individuales (uso del modelo de Kothari et al., 2005), la evidencia indica que dichos ajustes por devengo no varían significativamente bajo IFRS respecto al periodo de aplicación de los GAAP rumanos.

Adicionalmente, la calidad contable en Francia, Grecia y Reino Unido con la entrada en vigencia de las IFRS, también ha sido objeto de análisis, estudios realizados por Zéghal, Chtourou y Sellami (2011) entre los años 2003 y 2006, Dimitropoulos, Asteriou, Kousenidis y Leventis (2013) para el periodo 2001-2008 e Iatridis (2010) entre el 2004 y 2005 respectivamente, han sugerido que la calidad de la información contable mejora con el uso de dichos estándares, dado que evidencian una disminución significativa en el nivel de manipulación del resultado respecto a la aplicación de los GAAP Locales de cada país. Estos hallazgos fueron conducidos utilizando el modelo propuesto por Kothari et al. (2005) para los dos primeros países en mención y el modelo básico de Jones (1991) para Reino Unido.

El modelo propuesto por Jones (1991) y sus posteriores modificaciones, también han sido empleados ampliamente en los países orientales, siendo Corea y China los países con mayor número de estudios reportados; para el caso Coreano, se ha documentado varios estudios sobre el cambio en la calidad contable por la aplicación voluntaria de las IFRS durante el periodo 2007-2010 (Kim, 2013), y por la aplicación obligatoria de las mismas (Kim, 2014), considerando para este último caso, un periodo máximo de análisis de 6 años, entre 2007 a 2012 (Kwon y Whan Oh, 2015) y entre 2008 a 2013 (Jong-Il, 2015); los análisis indican que la magnitud de los ajustes por devengo discrecionales, aumenta durante el periodo voluntario de aplicación de las IFRS, mientras que disminuye con la aplicación obligatoria de las mismas. Todos estos resultados fueron obtenidos empleando el modelo propuesto por Dechow et al. (1995) excepto Jong-Il (2015), quien emplea el modelo propuesto por Kothari et al. (2005).

Los hallazgos reportados para las empresas listadas en China respecto al cambio en la calidad contable con la entrada obligatoria de las IFRS, sugieren resultados diversos, Zhang, Uchida y Bu (2013), realizaron un estudio entre los años 2001 a 2010 utilizando el modelo de Kothari et al. (2005), estos autores indican un aumento significativo en el nivel de manipulación del resultado, mientras que Ho, Liao y Taylor (2015), sugieren todo lo contrario, una menor probabilidad en el uso de los ajustes por devengo discrecionales (menor nivel de manipulación del resultado), al utilizar el modelo propuesto por Dechow et al. (1995) entre los años 2002 y 2011. Nuevamente se advierte sobre la diversidad en los resultados respecto a la entrada en vigencia de las IFRS para otras economías orientales, por ejemplo para las compañías cotizadas en el mercado de capitales de la India, se reporta un incremento en el nivel de manipulación del resultado respecto a aquellas compañías no adoptantes durante el año 2010 (Rudra y Bhattacharjee, 2012); por su parte Adibah Wan Ismail, Anuar Kamarudin, Van Zijl y Dunstan (2013), sugieren una disminución en dicho nivel de manipulación para las empresas de Malasia, comparando 3 años antes y 3 años después de la aplicación de dichos estándares; Finalmente, Bryce, Ali y Mather (2015), indican que la aplicación de las IFRS no tiene efectos significativos en la calidad de la información contable australiana, analizando conjuntamente los años comprendidos entre el 2003 y 2008; todos estos estudios han sido conducidos bajo el modelo propuesto por Dechow et al. (1995).

Durante la última década, la entrada obligatoria de las IFRS en casi todos los países del 
continente americano, ha traído consigo diversos estudios sobre la calidad contable por parte de académicos y organismos reguladores en la materia, México y Brasil son los países latinos sobre los que se concentra la mayoría de estos estudios; inicialmente Conesa, Manzano y Sánchez (2011), al analizar la adaptación de los GAAP mexicanos hacia los estándares IFRS, sugieren una disminución en el nivel de manipulación del resultado bajo un amplio periodo de análisis (1997 a 2009), sin embargo, Palacios Manzano y Martínez Conesa (2014) orientando su análisis entre los años 1997 y 2008, reportan que los ajustes por devengo discrecionales no disminuyen con el uso de las IFRS (no disminuye el nivel de manipulación del resultado) para aquellas compañías no financieras mexicanas listadas en el NYSE; estos estudios fueron conducidos utilizando los modelos de Jones (1991) y Dechow et al. (1995).

Para el caso de las empresas listadas en el mercado de capitales Brasileño, Pelucio-Grecco, Geron, Grecco y Lima (2014), direccionan su estudio durante los periodos 2006-2011 utilizando una gran variedad de modelos para testar su hipótesis (uso de los modelos de Dechow et al., 1995; Jones, 1991; Kothari et al., 2005), sus resultados indican que la transición a IFRS restringe el nivel de manipulación del resultado después de completar la implementación respecto a los periodos previos a dichas normas, sin embargo, Santana, Timm, Costa y Zoboli (2014), al comparar la magnitud de los ajustes por devengo discrecionales (usando los modelos de Dechow et al., 1995; Jones, 1991) entre las empresas cotizadas en Brasil y Chile respecto Reino Unido y Australia, y respecto a Francia y Alemania, sugieren que las empresas latinas reportan un menor nivel de calidad contable durante los años 2011 y 2012. El reporte de estudios de la calidad contable en el continente americano finaliza con Canadá, siendo Leung (2015), quien conduce un estudio sobre los años 2005 a 2014, al considerar los efectos de la aplicación obligatoria de las IFRS en el territorio canadiense, obteniendo evidencia que le sugiere un aumento en la calidad contable dado una disminución en el nivel de ajustes por devengo discrecionales (uso de los modelos de Dechow et al., 1995; Kothari et al., 2005).

En general, la gran mayoría de los estudios multi-país sobre la calidad contable a partir del uso de las IFRS, corresponden a los países pertenecientes a la Unión Europea, dichos estudios son aplicados sobre las empresas cotizadas en los mercados de estos países, de los cuales se obtienen resultados muy diversos respecto a los efectos de la aplicación de dichos estándares en el nivel de manipulación del resultado; estudios como los direccionados por Chen, Tang, Jiang y Lin (2010) (utilizando el modelo de jones y sus sucesivas modificaciones indicadas hasta ahora) y por Zeghal, Chtourou y Fourati (2012) (utilizando el modelo de Dechow et al., 1995 entre los años 2001 a 2007), ambos aplicados sobre 15 países europeos, indican que la calidad contable aumenta dado una disminución en la magnitud de los ajustes por devengo discrecionales durante los periodos de post-adopción; adicionalmente, Aussenegg, Inwinkl y Schneider (2008), aplican el mismo estudio sobre 17 países europeos bajo el modelo propuesto por Dechow et al. (1995) e incorporando algunas variaciones, si bien sus resultados apuntan a una disminución en el nivel de manipulación del resultado, los hallazgos sugieren que dicho nivel de manipulación no varía significativamente para aquellos países con influencia anglosajona (Reino Unido e Irlanda) y escandinava (países del norte), dado que antes de la aplicación obligatoria de las IFRS, estos países reportaban una menor manipulación del resultado respecto a los demás países europeos.

Otros estudios aplicados sobre los países pertenecientes a la Unión Europea, sugieren que el uso obligatorio de las IFRS no tiene impacto significativo en la magnitud de los ajustes por devengo discrecionales, siendo ejemplo de esto, los estudios conducidos durante los años 2000 a 
2010 por Doukakis (2014) sobre 22 países europeos (uso del modelo de Dechow et al., 1995) y por Gray, Kang, Lin y Tang (2015) sobre 14 de estos países (uso de los modelos de Jones, 1991; Kothari et al., 2005). En correspondencia con lo anterior, Callao y Jarne (2010), analizan la aplicación de las IFRS sobre 11 economías europeas entre los años 2003 a 2006 (uso del modelo propuesto por Larcker y Richardson, 2004), sus resultados indican que el uso obligatorio de las IFRS surte efectos negativos sobre el nivel de manipulación del resultado, dado evidencia que sugiere un incremento significativo en los ajustes por devengo discrecionales.

Los demás estudios multi-país reportados a la fecha, aparte de incluir países pertenecientes a la unión europea, incorporan además países orientales y latinoamericanos, todos ellos arribando hacia una misma conclusión, las IFRS no mejoran la calidad de la información contable para los países analizados en sus respectivas muestras, o al menos por si solas; por el contrario, tienden a disminuir la calidad durante el periodo de aplicación de dichos estándares; estudios que sostienen esta afirmación son los direccionados por Sang-Ae, Nam-Ryoung y Sang-Bum (2011) entre los años 2001 a 2008 (uso del modelo de Kothari et al., 2005), Ahmed, Neel y Wang (2013) para 20 economías que adoptaron las IFRS en el año 2005 relativo a aquellas que no lo hicieron, y finalmente Houqe, Van Zijl, Dunstan y Karim (2012) sobre 46 países entre los años 1998 y 2007 (ambos utilizando el modelo de Dechow et al., 1995).

De acuerdo con los anteriores hallazgos a nivel mundial y en especial con aquellos obtenidos para las principales economías del continente americano consideradas individualmente, se postulan las siguientes hipótesis:

Hipótesis 1 (H1). La magnitud absoluta de los ajustes por devengo discrecionales disminuye significativamente durante el periodo de aplicación de IFRS (menor manipulación del resultado) respecto al periodo de pre-IFRS para las empresas latinoamericanas y del Caribe.

Hipótesis 2 (H2). Existe una relación estadísticamente negativa entre la magnitud absoluta de los ajustes por devengo discrecionales y los periodos de aplicación de las IFRS por parte de las empresas latinoamericanas y del Caribe (impacto de las IFRS en el nivel de manipulación del resultado).

\section{Metodología}

Investigaciones previas utilizan la magnitud de los ajustes por devengo discrecionales como indicador de medición de la manipulación del resultado, y este a su vez como proxy de medición de la calidad del reporte financiero.

\section{Magnitud de los ajustes por devengo discrecionales}

El punto de partida en investigaciones previas para evaluar la manipulación del resultado a partir de la magnitud de los ajustes por devengo, es a través del modelo propuesto por Jones (1991); en su momento, en esta investigación se utilizó un modelo de regresión lineal a través de series temporales, el cual propone la capacidad de los cambios en las ventas y la magnitud bruta de la propiedad, planta y equipo en explicar el importe de los ajustes por devengo totales. El modelo básico de Jones, como se ha denominado comúnmente en la literatura, ha sido el punto de partida para que investigaciones posteriores modifiquen dicho modelo para testar sus resultados, por tanto se utiliza el modelo básico de Jones y sus sucesivas modificaciones para analizar el nivel de manipulación del resultado.

Según lo indicado por Osma, Enguídanos, Clemente y Lara (2004), al utilizarse un modelo de serie temporal se requiere un mínimo de observaciones por empresa a lo largo de varios 
periodos continuos, y esto supone una reducción de la muestra de observaciones que no cumplan con este requisito. Por tanto DeFond y Jiambalvo (1994), han propuesto estimar el modelo utilizando los datos bajo un estudio de corte transversal (cross-sectional), agrupando las empresas por país e industria en cada año, y esto a parte de disminuir el efecto que se genera si se utilizase los datos organizados en series de tiempo, también asume que los coeficientes que se generan no son constantes por empresa a través del tiempo, sino que se asume los coeficientes constantes en las empresas pertenecientes a la misma industria y país para un periodo determinado.

El modelo básico de Jones (1991), se basa en la magnitud absoluta de los ajustes por devengo discrecionales o anormales bajo un análisis de corte transversal (the magnitude of crosssectional absolute discretionary accruals). Este indicador se basa en la estimación de ajustes por devengo discrecionales, donde son definidos como el resultado de los ajustes por devengo totales observados menos la estimación de los ajustes por devengo normales (no discrecionales) a través del modelo de Jones (1991), es decir, los ajustes por devengo discrecionales son los residuos de la regresión. Dichos componentes, discrecional y no discrecional se determinan a través de cinco (5) modelos de ajustes por devengo discrecionales, los cuales son comúnmente utilizados en investigaciones previas, sin embargo es poco frecuente que todos estos modelos sean utilizados conjuntamente en una misma investigación: el modelo básico de Jones, el modelo de Jones modificado, el modelo de Jones adaptado, el modelo de Jones modificado con el ratio de valor en libros sobre valor de mercado del patrimonio (book to market ratio) y el Cash Flow operativo, y el modelo de Jones modificado con el ROA del año corriente (Dechow et al., 2003; Dechow et al., 1995; Kothari et al., 2005; Larcker y Richardson, 2004). Se espera que a menor magnitud absoluta de los ajustes por devengo discrecionales, menor es el nivel de manipulación del resultado, lo que es lo mismo, mayor calidad contable.

Debido a que los ajustes por devengo discrecionales pueden resultar en aumento o disminución del resultado con dirección hacia una ganancia objetivo, se considera la magnitud absoluta de dichos ajustes para valorar el grado de manipulación del resultado. Cada modelo se aplica en cada periodo de análisis (GAAP Local e IFRS) y para cada industria; en primer lugar se estiman los ajustes por devengo en cada uno de los cinco modelos, seguidamente los coeficientes estimados de estos modelos de regresión se utilizan para calcular los ajustes por devengo discrecionales (ajustes por devengo totales observados menos los ajustes por devengo no discrecionales).

Modelo de Jones (en corte transversal): En el modelo de expectativas propuesto por Jones (1991), se utilizan los ajustes por devengo a corto y a largo plazo, se trata de regresar los ajustes por devengo totales observados sobre el cambio en las ventas, el cual modeliza el componente normal de ajustes por devengo del corto plazo, y el nivel del inmovilizado material bruto (propiedad, planta y equipo), el cual modeliza el componente no discrecional de los ajustes del largo plazo por gasto depreciación y amortización.

De acuerdo con Osma et al. (2004), dependiendo de las características de los países, se prefiere utilizar ambos componentes en las investigaciones, el componente normal de los ajustes por devengo del corto y del largo plazo, mientras que en otras prefieren simplemente el componente normal del corto plazo; esta elección se basa en el grado de visibilidad que los usuarios de la información ejercen sobre las cuentas, por tanto si en un país, uno de los principales financiadores de las compañías es el mercado de capitales y se tiene una estructura 
de propiedad dispersa, posiblemente se prescinda en utilizar el componente normal de los ajustes por devengo del largo plazo; en caso contrario, seguramente no se prescinda de estos. Por lo anterior, en esta investigación se utiliza ambos componentes.

$$
\frac{A C C_{i t}}{A_{i t-1}}=\frac{\alpha 1}{A_{i t-1}}+\beta_{1} \frac{\Delta R E V_{i t}}{A_{i t-1}}+\beta_{2} \frac{P P E_{i t}}{A_{i t-1}}+\varepsilon_{i t}
$$

En donde:

$A C C=$ son los ajustes por devengo totales observados, definido como la utilidad neta operativa (antes de partidas extraordinarias) menos el Cash Flow Operativo del periodo; $A=$ representa el activo total; $R E V=$ representa el cambio en las ventas del periodo; $P P E=$ es el importe bruto de la propiedad, planta y equipo; $E=$ representa el término de error del modelo; Los subíndices i y t representan a la empresa y el periodo respectivamente. Todas las variables del modelo se dividen por el activo total del inicio del periodo para evitar posibles problemas de heterocedasticidad.

Los coeficientes procedentes del modelo estimado anterior, se utilizan para calcular los ajustes por devengo discrecionales

$$
D A C C_{i t}=\left(\frac{A C C_{i t}}{A_{i t-1}}\right)-\left(\frac{\alpha 1}{A_{i t-1}}+\beta_{1} \frac{\Delta R E V_{i t}}{A_{i t-1}}+\beta_{2} \frac{P P E_{i t}}{A_{i t-1}}\right)
$$

En donde:

$D A C C=$ son los ajustes por devengo discrecionales; los demás componentes son definidos arriba.

Modelo de Jones modificado (En corte transversal): Dechow et al. (1995) agrega que el modelo de expectativas de Jones se basa en que las ventas no presentan ninguna discrecionalidad, es inevitable que la discrecionalidad estimada por su modelo no presente errores, debido a que la dirección puede manipular las ganancias a través de las ventas. Por tanto Dechow et al. (1995) añade la variable cambio en las cuentas por cobrar, argumentando que esto posiblemente eliminaría la imprecisión presentada en el modelo básico de Jones. Por lo anterior, en este modelo se estiman los coeficientes desde la ecuación (1) bajo un modelo de mínimos cuadrados ordinarios (MCO) y dichos coeficientes se emplean en la siguiente ecuación para estimar los ajustes por devengo discrecionales.

$$
D A C C_{i t}=\left(\frac{A C C_{i t}}{A_{i t-1}}\right)-\left(\frac{\alpha 1}{A_{i t-1}}+\beta_{1}\left[\frac{\Delta R E V_{i t}}{A_{i t-1}}-\frac{\Delta R E C_{i t}}{A_{i t-1}}\right]+\beta_{2} \frac{P P E_{i t}}{A_{i t-1}}\right)
$$

En donde:

$\Delta R E C_{i t}=$ Es el cambio en las cuentas por cobrar; las demás variables se describen arriba.

Modelo de Jones adaptado (en corte transversal): El modelo anterior ha recibido sus críticas a nivel académico, como lo ha manifestado Dechow et al. (2003), quienes agregan 
que el modelo de Jones modificado asume que todas las ventas a crédito en cada periodo son ajustes discrecionales, por tanto induce a una correlación positiva entre los ajustes por devengo discrecionales y el crecimiento en las ventas en cada periodo; por lo anterior se incluye una porción inesperada del cambio en las cuentas por cobrar en los ajustes por devengo discrecionales. Los coeficientes estimados se siguen calculando de acuerdo a la ecuación 1, y dichos coeficientes deben ser utilizados en la siguiente ecuación para calcular los ajustes por devengo discrecionales:

$$
D A C C_{i t}=\left(\frac{A C C_{i t}}{A_{i t-1}}\right)-\left(\frac{\alpha 1}{A_{i t-1}}+\beta_{1}\left[(1+k) \frac{\Delta R E V_{i t}}{A_{i t-1}}-\frac{\Delta R E C_{i t}}{A_{i t-1}}\right]+\beta_{2} \frac{P P E_{i t}}{A_{i t-1}}\right)
$$

En donde:

El coeficiente $k$, es estimado desde la siguiente ecuación por periodo y en cada industria, lo cual captura el cambio esperado en las cuentas por cobrar por un determinado cambio en las ventas.

$$
\Delta R E C_{i t}=\left(\alpha+k_{1} \Delta R E V_{i t}+\varepsilon_{i t}\right)
$$

Modelo de Jones modificado con el ratio valor en libros sobre valor de mercado del patrimonio y cash flow operativo (En corte transversal): Según Larcker y Richardson (2004), se añaden dos variables al modelo de Jones modificado con el objeto de mitigar la magnitud del error contenido en los ajustes por devengo discrecionales estimados, por tanto agregan estas variables de control las cuales son el ratio book to market y el Cash Flow operativo. En la ecuación (6) se hallan los coeficientes estimados por industria en cada periodo y estos coeficientes se aplican en la ecuación (7) para hallar los ajustes por devengo discrecionales.

$$
\begin{gathered}
\frac{A C C_{i t}}{A_{i t-1}}=\frac{\alpha 1}{A_{i t-1}}+\beta_{1} \frac{\Delta R E V_{i t}}{A_{i t-1}}+\beta_{2} \frac{P P E_{i t}}{A_{i t-1}}+\beta_{3} B M_{i t}+\beta_{4} \frac{C F O_{i t}}{A_{i t-1}}+\varepsilon_{i t} \\
D A C C_{i t}=\left(\frac{A C C_{i t}}{A_{i t-1}}\right)-\left(\frac{\alpha 1}{A_{i t-1}}+\beta_{1}\left[\frac{\Delta R E V_{i t}}{A_{i t-1}}-\frac{\Delta R E C_{i t}}{A_{i t-1}}\right]+\beta_{2} \frac{P P E_{i t}}{A_{i t-1}}+\beta_{3} B M_{i t}+\beta_{4} \frac{C F O_{i t}}{A_{i t-1}}\right)
\end{gathered}
$$

En donde:

$B M=$ es el ratio del valor en libros sobre el valor de mercado del patrimonio para la empresa i en el año t; $C F O=$ Es el Cash Flow desde la operación escalado por el total de activos; las demás variables fueron indicadas arriba.

Modelo de Jones modificado con el ROA del año corriente (En corte transversal): La adición al modelo de Jones modificado fue desarrollado por Kothari et al. (2005), quienes argumentan que los ajustes por devengo de las empresas que tienen un desempeño (rentabilidad) inusual se espera que sea diferente de cero, por esto el desempeño de la compañía y los ajustes por devengo están correlacionados. De acuerdo a esto, Kothari et al. (2005) añade una nueva 
variable al modelo de Jones modificado, el ROA del año actual con el objeto de controlar el desempeño. Por tanto desde la ecuación (8), se estiman los coeficientes los cuales se utilizan en el modelo contenido en la ecuación (9), para hallar los ajustes por devengo discrecionales.

$$
\begin{aligned}
& \frac{A C C_{i t}}{A_{i t-1}}=\frac{\alpha 1}{A_{i t-1}}+\beta_{1} \frac{\Delta R E V_{i t}}{A_{i t-1}}+\beta_{2} \frac{P P E_{i t}}{A_{i t-1}}+\beta_{3} R O A_{i t}+\varepsilon_{i t} \\
& D A C C_{i t}=\left(\frac{A C C_{i t}}{A_{i t-1}}\right)-\left(\frac{\alpha 1}{A_{i t-1}}+\beta_{1}\left[\frac{\Delta R E V_{i t}}{A_{i t-1}}-\frac{\Delta R E C_{i t}}{A_{i t-1}}\right]+\beta_{2} \frac{P P E_{i t}}{A_{i t-1}}+\beta_{3} R O A_{i t}\right)
\end{aligned}
$$

En donde:

$R O A=$ Es la rentabilidad de los activos para la empresa i en el año t; las demás variables fueron definidas arriba.

Los modelos contenidos en las ecuaciones del 1 al 9 son regresados por el modelo de mínimos cuadrados ordinarios (MCO) clasificados por marco normativo y por tipo de industria para atender a lo indicado por Osma et al. (2004), en lo relacionado con un estudio en corte transversal (Cross-sectional).

Modelos para relacionar los efectos de los incentivos de la gerencia sobre los ajustes por devengo discrecionales

Siguiendo a Chen et al. (2010) y Van-Tendeloo y Vanstraelen (2005), la magnitud absoluta de los ajustes por devengo discrecionales, obtenida en cada uno de los modelos anteriores, se regresa individualmente sobre un conjunto de variables de control, las cuales agrupan las características económicas de cada compañía y los efectos de cada país en los que opera la misma; dichas características sugieren posiblemente, los incentivos que tendría cada una de ellas para emplear el uso de los ajustes por devengo discrecionales, como medida de la manipulación del resultado. La siguiente ecuación estima el efecto de la adopción de las IFRS e incentivos de la gerencia y país sobre la magnitud absoluta de los ajustes por devengo discrecionales.

$$
\begin{aligned}
\mid D_{A C C_{i t} \mid=\alpha} & +\beta_{1} \text { POST }_{i t}+\beta_{2} \text { SIZE }_{i t}+\beta_{3} \text { LEV }_{i t}+\beta_{4} \text { GROWTH }_{i t}+\beta_{5} \text { TURN }_{i t}+\beta_{6} \text { CFO }_{i t}+\beta_{7} \text { AUD }_{i t} \\
& +\beta_{8} \text { NUMEX }_{i t}+\beta_{9} \text { LOSS }_{i t}+\beta_{10} \text { XLIST }_{i t}+\sum_{l=1}^{24} \beta_{l+10} \text { Country }_{i}+\varepsilon_{i t}
\end{aligned}
$$

En donde:

$\left|D A C C_{i t}\right|$ Es la magnitud absoluta de los ajustes por devengo discrecionales hallados por medio de los 5 modelos de Jones definiéndolos como sigue: $\left|D A C C_{i t}\right| 1=$ es la magnitud absoluta de los ajustes por devengo discrecionales en el modelo básico de Jones; $\left|D A C C_{i t}\right| 2=$ es la magnitud absoluta de los ajustes por devengo discrecionales en el modelo de Jones modificado; $\left|D A C C_{i t}\right| 3=$ es la magnitud absoluta de los ajustes por devengo discrecionales en el modelo de Jones Adaptado; $\left|D A C C_{i t}\right| 4=$ es la magnitud absoluta de los ajustes por devengo discrecionales 
en el modelo de Jones modificado con el ratio book to market y el Cash Flow operativo; $\left|D A C C_{i t}\right| 5=$ es la magnitud absoluta de los ajustes por devengo discrecionales en el modelo de Jones modificado con el ROA del año corriente para la empresa $i$ en el año $t$; POST $=$ es un indicador variable, toma valores de 1 en los periodos en los cuales las empresas aplicar IFRS y 0 en otro caso, un coeficiente significativamente negativo en POST, indica que las empresas tienen menor magnitud absoluta de ajustes por devengo discrecionales en el periodo de postadopción que en el periodo de pre-adopción de las IFRS. SIZE= es el logaritmo natural al final del año del total de activos e indica el tamaño de la compañía; $L E V=$ pasivos totales al final del periodo dividido por el valor en libros del patrimonio al final del periodo. Indica el nivel de endeudamiento; GROWTH= cambio porcentual en las ventas; TURN= ventas dividido por el total de activos al final del año. Indica la rotación o gestión en los activos; $C F O=$ Cash Flow operativo al final del año dividido por el total de activos al final del año; $A U D=$ es una variable dicótoma que toma valor de uno (1) si las empresas son auditadas por alguna de las Big4 (PWC, KPMG, EY o DELOITTE) y cero (0) en otro caso; NUMEX= número de bolsas en las cuales cotiza cada compañía; $L O S S=$ es un indicador variable que toma valor de 1 para las observaciones con utilidad neta menor que cero, y 0 en otro caso; XLIST = variable dicótoma que toma valor de uno (1) si la empresa cotiza en algún mercado accionario de los Estados Unidos, y dicho mercado no es su bolsa principal de cotización; Country= es un indicador variable, siendo Argentina usada como referencia (Benchmark). El total de países en la muestra es 25, para efectos del modelo econométrico se establecen 24 variables, para evitar el problema de la multicolinealidad perfecta, esto aplica para calcular los modelos desde el 1 al 3 y el modelo 5; puesto que para el cálculo del modelo 4 la muestra sólo representa a 18 países, por tanto en el modelo econométrico se incluirán 17 países para evitar problemas de multicolinealidad perfecta. En todas las variables, exceptuando las variables binarias, se controla los efectos de los valores outliers (winsorized) en los percentiles 5 y 95.

\section{Muestra y distribución}

La muestra está conformada por las empresas domiciliadas en los países latinoamericanos y del Caribe que reporten información contable y bursátil disponible en la base de datos de información financiera ORBIS (ORBIS en adelante) y que coticen o hayan cotizado para todos o alguno de los periodos desde el 2006 hasta el 2014. La muestra incluye aquellas empresas que durante este periodo hayan aplicado las IFRS independientemente si fue voluntaria u oficial su adopción, ORBIS identifica el marco normativo en el cual cada compañía reporta anualmente sus estados financieros. Sólo se incluyen aquellas empresas que presenten datos disponibles tanto en su normativa local (GAAP Local) para su periodo de pre-adopción e IFRS para su periodo de post-adopción. Se agrupan los datos bajo un estudio de corte transversal comparando el periodo de pre y post-adopción de IFRS, ambos con referencia al mismo periodo de análisis (2006-2014), lo anterior para evitar cambios en la calidad contable atribuibles a las condiciones económicas de diferentes años.

La Tabla 1, muestra a detalle la selección de la muestra, iniciando a partir de todas las compañías cotizadas y domiciliadas en América Latina y el Caribe, luego se filtraron aquellas compañías con información continua disponible menor a tres años, al igual que aquellas firmas que durante el periodo de análisis (2006 a 2014) no reportaron información bajo IFRS; obteniéndose así una muestra general de 925 empresas, 854 pertenecientes a América Latina y el resto a países del Caribe. De esta muestra general de empresas y del total del número 
de años analizados en los cuales las empresas tienen información disponible se obtienen las observaciones para el análisis, alcanzando un total de 5978 observaciones, 2133 (35.7\%) observaciones pertenecientes al periodo de Pre-adopción (GAAP Local) y las restantes 3845 $(64.3 \%)$ pertenecen al periodo de Post-adopción (IFRS). Partiendo de este total se filtra las observaciones que no presenten la información necesaria para calcular cada una de las variables de los distintos modelos definidos en la metodología. Por tanto para los modelos básico de Jones, Jones ajustado y Jones adaptado (Muestra A) se obtiene una muestra final de 5490 observaciones; para el modelo de Jones modificado con el ratio valor en libros sobre valor de mercado del patrimonio y cash flow operativo (Muestra B) se obtiene un total de 4020 observaciones, y para el último modelo utilizado, el modelo de Jones modificado con el ROA del año corriente, lo integran finalmente 5436 observaciones.

Tabla 1

Selección de la muestra

\begin{tabular}{|c|c|c|c|}
\hline \multicolumn{4}{|c|}{ Selección de la muestra general (clasificado por número de compañías) } \\
\hline & $\begin{array}{l}\text { América } \\
\text { Latina }\end{array}$ & E1 Caribe & Total \\
\hline $\begin{array}{l}\text { Compañías clasificadas como activas en ORIBS, cotizadas o que } \\
\text { cotizaron durante los años } 2006 \text { a 2014, con domicilio en } \\
\text { América Latina o el caribe. }\end{array}$ & 1515 & 2292 & 3807 \\
\hline $\begin{array}{l}\text { Compañías no cotizadas en los mercados de valores } \\
\text { latinoamericanos o del Caribe. }\end{array}$ & 0 & (2 147) & $(2147)$ \\
\hline $\begin{array}{l}\text { Compañías con información no disponible (con menos de } 3 \text { años } \\
\text { continuos de información contable) }\end{array}$ & $(264)$ & (28) & $(292)$ \\
\hline $\begin{array}{l}\text { Compañías que no reportan información bajo IFRS durante los } \\
\text { años } 2006 \text { a } 2014\end{array}$ & $(397)$ & (46) & $(443)$ \\
\hline Total muestra general (clasificado por número de compañías) & 854 & 71 & 925 \\
\hline \multirow{2}{*}{$\begin{array}{l}\text { Total muestra general (clasificado por número de observaciones } \\
\text { y marco normativo contable) }\end{array}$} & GAAP Local & IFRS & Total \\
\hline & 2133 & 3845 & 5978 \\
\hline
\end{tabular}

Selección de la muestra para cada modelo de ajustes por devengo a partir de la muestra general (clasificado por número de observaciones y marco normativo contable)

Selección de la muestra A: modelos de ajustes por devengo 1 (modelo básico de Jones), 2 (modelo de Jones ajustado), 3 (modelo de Jones adaptado)

\begin{tabular}{llll}
\hline Marco Normativo & GAAP Local & IFRS & Total \\
\hline $\begin{array}{l}\text { Número de observaciones desde la muestra general (clasificado } \\
\text { por número de observaciones y marco normativo contable) }\end{array}$ & 2133 & 3845 & 5978 \\
$\begin{array}{l}\text { Datos perdidos para las variables de medición de los ajustes por } \\
\text { devengo modelos 1, } 2 \text { y } 3\end{array}$ & $(156)$ & & \\
$\begin{array}{l}\text { Total observaciones para la muestra A (corresponde a } 876 \\
\text { Empresas) }\end{array}$ & 1977 & 3513 & 5490
\end{tabular}

Empresas) 
http://dx.doi.org/10.22201/fca.24488410e.2018.1233

Selección de la muestra C: modelo de ajustes por devengo 5 (modelo de Jones modificado con ROA del año corriente)

\begin{tabular}{llll}
\hline Marco Normativo & GAAP Local & IFRS & Total \\
\hline $\begin{array}{l}\text { Número de observaciones desde la muestra general (clasificado } \\
\text { por número de observaciones y marco normativo contable) }\end{array}$ & 2133 & 3845 & 5978 \\
$\begin{array}{l}\text { Datos perdidos para las variables de medición de los ajustes por } \\
\text { devengo modelo 5 }\end{array}$ & (169) & & (542) \\
$\begin{array}{l}\text { Total observaciones para la muestra C (corresponde a } 870 \\
\text { Empresas) }\end{array}$ & 1964 & 3472 & 5436 \\
\hline Fuente: elaboración propia
\end{tabular}

En la Tabla 2 se presenta la distribución de las observaciones por país, mientras que la Tabla 3 las presenta por Industria. En la Tabla 2, para las muestras A y C (muestra B) participan observaciones desde 25 países (18 países) de Latinoamérica y el Caribe, de los cuales el 33\% (35\%) de las observaciones pertenecen a Brasil, seguidos de un 19\% (21\%) pertenecientes a Chile; el 12\% (13\%) de las observaciones pertenecen a México, mientras que el 9\% (8\%) pertenece a Perú, seguido de un 7\% (7\%) perteneciente a Argentina.

Table 2

Distribución de la muestra por país

\begin{tabular}{|c|c|c|c|c|c|c|}
\hline \multirow[t]{2}{*}{ País } & \multicolumn{2}{|c|}{ MUESTRA A } & \multicolumn{2}{|c|}{ MUESTRA B } & \multicolumn{2}{|c|}{ MUESTRA C } \\
\hline & Núm. Obs. & $\%$ Obs. & Núm. Obs. & $\%$ Obs. & Núm. Obs. & $\%$ Obs. \\
\hline Argentina & 390 & 0.07 & 293 & 0.07 & 389 & 0.07 \\
\hline Barbados & 41 & 0.01 & 40 & 0.01 & 41 & 0.01 \\
\hline Bermuda & 38 & 0.01 & 27 & 0.01 & 38 & 0.01 \\
\hline Bolivia & 96 & 0.02 & 40 & 0.01 & 96 & 0.02 \\
\hline Brazil & 1,822 & 0.33 & 1,424 & 0.35 & 1,790 & 0.33 \\
\hline Bahamas & 27 & 0.00 & 27 & 0.01 & 27 & 0.00 \\
\hline Chile & 1,051 & 0.19 & 840 & 0.21 & 1,044 & 0.19 \\
\hline Colombia & 158 & 0.03 & 88 & 0.02 & 158 & 0.03 \\
\hline Costa Rica & 27 & 0.00 & 15 & 0.00 & 27 & 0.00 \\
\hline Dominica & 8 & 0.00 & 0 & 0.00 & 8 & 0.00 \\
\hline Ecuador & 123 & 0.02 & 72 & 0.02 & 123 & 0.02 \\
\hline Granada & 7 & 0.00 & 0 & 0.00 & 7 & 0.00 \\
\hline Guatemala & 9 & 0.00 & 0 & 0.00 & 9 & 0.00 \\
\hline Guyana & 14 & 0.00 & 0 & 0.00 & 8 & 0.00 \\
\hline Jamaica & 129 & 0.02 & 120 & 0.03 & 128 & 0.02 \\
\hline San Cristobal & 14 & 0.00 & 0 & 0.00 & 14 & 0.00 \\
\hline Caiman Islands & 4 & 0.00 & 0 & 0.00 & 4 & 0.00 \\
\hline Mexico & 649 & 0.12 & 508 & 0.13 & 649 & 0.12 \\
\hline Panama & 64 & 0.01 & 50 & 0.01 & 64 & 0.01 \\
\hline Peru & 479 & 0.09 & 307 & 0.08 & 479 & 0.09 \\
\hline Paraguay & 123 & 0.02 & 0 & 0.00 & 123 & 0.02 \\
\hline El Salvador & 24 & 0.00 & 8 & 0.00 & 24 & 0.00 \\
\hline Trinidad and Tobago & 101 & 0.02 & 100 & 0.02 & 94 & 0.02 \\
\hline Uruguay & 26 & 0.00 & 1 & 0.00 & 26 & 0.00 \\
\hline Venezuela & 66 & 0.01 & 60 & 0.01 & 66 & 0.01 \\
\hline Total & 5,490 & 1 & 4,020 & 1 & 5,436 & 1 \\
\hline
\end{tabular}

Fuente: elaboración propia

Las observaciones reportadas en la Tabla 2, con porcentaje de participación del $(0.00)$ y con número de observaciones mayor a 0 , presentan una participación menor al $1 \%$ del total de cada muestra. 
En la Tabla 3 se observa que para las muestras A y C (muestra B) participan observaciones pertenecientes a 18 industrias (18 industrias), de las cuales el $36 \%(40 \%)$ pertenecen a la industria manufacturera, el $12 \%(11 \%)$ a la industria eléctrica, seguido del comercio al por mayor y por menor y de la industria financiera representado en un $8 \%(8 \%)$ y $7 \%(7 \%)$, respectivamente.

Table 3

Distribución de la muestra por Industria

\begin{tabular}{lrrrrrr}
\hline \multicolumn{1}{c}{ Industria } & \multicolumn{2}{c}{ MUESTRA A } & \multicolumn{2}{c}{ MUESTRA B } & \multicolumn{2}{c}{ MUESTRA C } \\
\cline { 2 - 7 } & Núm. Obs. \% Obs. & Núm. Obs. & \% Obs. & Núm. Obs. \% Obs. \\
\hline Agricultura, ganadería y pesca & 297 & 0.05 & 174 & 0.04 & 297 & 0.05 \\
Industrias extractivas & 175 & 0.03 & 132 & 0.03 & 172 & 0.03 \\
Industria manufacturera & 1,980 & 0.36 & 1,594 & 0.40 & 1,949 & 0.36 \\
Suministro de energía eléctrica & 678 & 0.12 & 435 & 0.11 & 677 & 0.12 \\
Suministro de agua y saneamiento & 68 & 0.01 & 40 & 0.01 & 68 & 0.01 \\
Construcción & 253 & 0.05 & 210 & 0.05 & 253 & 0.05 \\
Comercio al por mayor y al por menor & 413 & 0.08 & 326 & 0.08 & 413 & 0.08 \\
Transporte y almacenamiento & 257 & 0.05 & 184 & 0.05 & 253 & 0.05 \\
Hostelería & 111 & 0.02 & 68 & 0.02 & 110 & 0.02 \\
Information et comunication & 266 & 0.05 & 179 & 0.04 & 265 & 0.05 \\
Actividades financieras y de seguros & 404 & 0.07 & 267 & 0.07 & 399 & 0.07 \\
Actividades inmobiliarias & 283 & 0.05 & 214 & 0.05 & 278 & 0.05 \\
Actividades profesionales, científicas y técnicas & 53 & 0.01 & 40 & 0.01 & 53 & 0.01 \\
Actividades administrativas & 26 & 0.00 & 13 & 0.00 & 26 \\
Educación & 40 & 0.01 & 9 & 0.00 & 40 & 0.00 \\
Actividades sanitarias y de servicios sociales & 49 & 0.01 & 48 & 0.01 & 49 & 0.01 \\
Actividades artísticas y recreativas & 94 & 0.02 & 71 & 0.02 & 92 & 0.01 \\
Otros servicios & 43 & 0.01 & 16 & 0.00 & 42 & 0.01 \\
Total & 5,490 & 1 & 4,020 & 1 & 5,436 & 1 \\
\hline Fut & & & & &
\end{tabular}

Fuente: elaboración propia

Las observaciones reportadas con porcentaje de participación del (0.00) presentan una participación menor al $1 \%$ del total de cada muestra.

\section{Resultados}

La Tabla 4 incluye el análisis estadístico de comparación de medias y medianas entre ambos periodos para las variables necesarias al estimar la magnitud absoluta de los ajustes por devengo discrecionales; los resultados demuestran que el total de ajustes por devengo observados (ACC), el total de activos al inicio de periodo (1/A), la variación en las ventas $(\triangle \mathrm{REV})$, la variación en las cuentas por cobrar $(\triangle \mathrm{REC})$, el importe de la propiedad, planta y equipo (PPE), el cash flow operativo (CFO) y la rentabilidad de los activos (ROA) son en media (mediana), significativamente menores (con un nivel de significancia del 1\%) durante el periodo de post-adopción respecto al periodo de pre-adopción, excepto para el total de ajustes por devengo observados y la rentabilidad de los activos, que resultan no tener diferencia estadísticamente significativa entre periodos. En cuanto al ratio valor en libros sobre valor de mercado del patrimonio (BM), resulta ser mayor en media, durante el periodo de aplicación de IFRS respecto al periodo de aplicación de las normas locales (GAAP Local), aunque dicha diferencia no resulta ser estadísticamente significativa.

Los resultados también demuestran un aumento estadísticamente significativo en media, para el tamaño de las empresas (SIZE) y la variación porcentual en las ventas (GROWHT), 
lo que repercute negativamente en la gestión de los activos (TURN), observándose en media (mediana), una disminución significativa para esta última variable durante el periodo de post-adopción respecto al periodo de pre-adopción; de igual forma se observa un aumento estadísticamente significativo en promedio, respecto al nivel de endeudamiento de las empresas (LEV) y en la contratación de los servicios de auditoría por parte de las Big4 (AUD); al tiempo se documenta un aumento en media, en cuanto al número de bolsas en las cuales cotiza las empresas (NUMEX) y, especialmente en la cotización de las acciones latinoamericanas y del Caribe en los mercados de capitales de los Estados Unidos (XLIST), durante el periodo de aplicación de IFRS respecto al periodo de pre-adopción. La variable (LOSS) a pesar de ser en media, menor durante el periodo de aplicación de IFRS, no presenta diferencia significativa entre ambos periodos, lo que posiblemente indicaría que durante el periodo de post-adopción, las empresas latinoamericanas y del Caribe continúan reconociendo las pérdidas en los estados financieros, con la misma frecuencia respecto al periodo de aplicación de las normas locales.

Table 4

Estadística descriptiva

Variables de control usadas al estimar la magnitud absoluta de los ajustes por devengo discrecionales (Discretionary Accruals)

\begin{tabular}{|c|c|c|c|c|c|c|c|c|c|}
\hline & \multicolumn{5}{|c|}{ Periodo Pre-adopción (GAAP Local) } & \multicolumn{4}{|c|}{ Periodo de Post-adopción (IFRS) } \\
\hline & $\begin{array}{c}\text { Comparación } \\
\text { varianza } \\
\end{array}$ & Obs. & Media & Mediana & $\begin{array}{c}\text { Desv. } \\
\text { Estándar }\end{array}$ & Obs. & Media & Mediana & $\begin{array}{c}\text { Desv. } \\
\text { Estándar }\end{array}$ \\
\hline $\mathrm{ACC}$ & $*$ & 1,977 & -0.027 & -0.023 & 0.104 & 3,510 & -0.026 & -0.024 & 0.078 \\
\hline 1/A & $*$ & 1,977 & 0.000 & 0.000 & 0.000 & 3,510 & $0.000 *$ & $0.000^{*}$ & 0.000 \\
\hline$\triangle \mathrm{REV}$ & $*$ & 1,977 & 0.118 & 0.063 & 0.219 & 3,510 & $0.019 *$ & $0.005 *$ & 0.129 \\
\hline$\triangle \mathrm{REC}$ & $*$ & 1,977 & 0.020 & 0.005 & 0.066 & 3,510 & $0.001 *$ & $0.000 *$ & 0.038 \\
\hline PPE & $*$ & 1,977 & 0.705 & 0.711 & 0.297 & 3,510 & $0.639 *$ & $0.654 *$ & 0.238 \\
\hline $\mathrm{BM}$ & $*$ & 1,332 & 1.371 & 0.875 & 1.546 & 2,688 & 1.458 & 0.862 & 1.674 \\
\hline $\mathrm{CFO}$ & $*$ & 1,977 & 0.122 & 0.108 & 0.144 & 3,510 & $0.108^{*}$ & $0.096 *$ & 0.113 \\
\hline ROA & $*$ & 1,964 & 5.875 & 5.395 & 8.540 & 3,472 & 5.839 & 5.315 & 8.009 \\
\hline SIZE & $*$ & 1,977 & 12.096 & 12.272 & 2.286 & 3,510 & $12.915 *$ & $13.012 *$ & 2.143 \\
\hline GROWTH & $*$ & 1,977 & 0.228 & 0.146 & 0.465 & 3,510 & $0.350 *$ & $0.016^{*}$ & 0.242 \\
\hline LEV & $*$ & 1,977 & 1.185 & 0.847 & 1.413 & 3,510 & $1.291 *$ & $0.916^{*}$ & 1.215 \\
\hline TURN & $*$ & 1,977 & 0.810 & 0.677 & 0.621 & 3,510 & $0.716^{*}$ & $0.628 *$ & 0.503 \\
\hline AUD & $*$ & 1,977 & 0.745 & 1.000 & 0.435 & 3,510 & $0.856^{*}$ & $1.000 *$ & 0.350 \\
\hline NUMEX & $*$ & 1,977 & 1.177 & 1.000 & 0.537 & 3,510 & $1.465^{*}$ & $1.000 *$ & 1.112 \\
\hline XLIST & $*$ & 1,977 & 0.012 & 0.000 & 0.109 & 3,510 & $0.071 *$ & $0.000 *$ & 0.257 \\
\hline LOSS & $*$ & 1,977 & 0.159 & 0.000 & 0.366 & 3,510 & 0.143 & 0.000 & 0.350 \\
\hline
\end{tabular}

Fuente: elaboración propia

$*, * *, * * *$ Indica diferencia significativa entre periodos con un nivel $\mathrm{p}<0.01, \mathrm{p}<0.05, \mathrm{p}<0.1$ respectivamente (prueba de dos colas). El supuesto de normalidad en la distribución de cada variable no es confirmado con el test de Shapiro-Wilk, este supuesto se relaja debido a que el tamaño de la muestra es lo suficientemente grande (superior a 30 observaciones), se asume normalidad de acuerdo al Teorema Central del Límite; para el análisis de significancia de medias entre ambos periodos para cada variable, previamente se realiza el test de igualdad de varianzas, dicho test arroja diferencia significativa entre ellas, por tanto se realiza el t-test de igualdad de medias con varianzas distintas. El test de igualdad de medianas, es comprobado para todas las variables a través del test de suma de rangos de Mann-Whitney-Wilcoxon excepto para la variable AUD, XLIST y LOSS, en la que se utiliza el test de comparación de proporciones basado en el test exacto de Fisher. 
La Tabla 5 proporciona un análisis univariable de la magnitud absoluta de los ajustes por devengo discrecionales, calculado bajo las 5 expresiones para los dos periodos de estudio, en todas ellas para el periodo de post-adopción, los ajustes por devengo son en media (mediana), significativamente menores respecto al periodo de pre-adopción, lo cual indica una disminución en el nivel de manipulación del resultado por parte de las empresas latinoamericanas y del Caribe y esto a su vez valida la hipótesis definida (H1).

Table 5

Comparación de la magnitud absoluta de los ajustes por devengo discrecionales (Discretionary Accruals) entre los periodos Local GAAP e IFRS

\begin{tabular}{|c|c|c|c|c|c|c|c|c|}
\hline \multirow[t]{2}{*}{ Medida } & \multicolumn{2}{|l|}{ Obs. } & \multicolumn{2}{|l|}{ Media } & \multirow[t]{2}{*}{ Diferencia } & \multicolumn{2}{|c|}{ Mediana } & \multirow[t]{2}{*}{ Diferencia } \\
\hline & GAAP Local & IFRS & GAAP Local & IFRS & & GAAP Local & IFRS & \\
\hline$\left|D A C C_{i j}\right| 1$ & 1,977 & 3,510 & 0.074 & 0.057 & $-0.016^{*}$ & 0.052 & 0.040 & $-0.011 *$ \\
\hline$\left|D A C C_{i t}^{t t}\right| 2$ & 1,977 & 3,510 & 0.074 & 0.057 & $-0.016^{*}$ & 0.052 & 0.040 & $-0.011 *$ \\
\hline$\left|D A C C_{i t}\right| 3$ & 1,977 & 3,510 & 0.074 & 0.057 & $-0.016^{*}$ & 0.053 & 0.041 & $-0.012 *$ \\
\hline$\left|D A C C_{i t}\right| 4$ & 1,332 & 2,688 & 0.054 & 0.041 & $-0.013^{*}$ & 0.041 & 0.030 & $-0.011 *$ \\
\hline$\left|D A C C_{i t}{ }_{i t}\right| 5$ & 1,964 & 3,472 & 0.072 & 0.056 & $-0.015^{*}$ & 0.052 & 0.040 & $-0.012 *$ \\
\hline
\end{tabular}

Fuente: elaboración propia

$*, * *, * * *$ Indica diferencia significativa entre periodos con un nivel $\mathrm{p}<0.01, \mathrm{p}<0.05, \mathrm{p}<0.1$, respectivamente (prueba de dos colas). El supuesto de normalidad en la distribución de cada variable no es confirmado con el test de Shapiro-Wilk, este supuesto se relaja debido a que el tamaño de la muestra es lo suficientemente grande (superior a 30 observaciones), se asume normalidad de acuerdo al Teorema Central del Límite; para el análisis de significancia de medias entre ambos periodos para cada variable, previamente se realiza el test de igualdad de varianzas, en su gran mayoría dicho test arroja diferencia significativa entre ellas, por tanto se realiza el t-test de igualdad de medias con varianzas distintas. El test de igualdad de medianas, es comprobado a través del test de suma de rangos de Mann-Whitney-Wilcoxon.

La Tabla 6 proporciona los resultados de la regresión de la magnitud absoluta de los ajustes por devengo discrecionales entre la adopción de las IFRS y las variables de control, bajo un modelo de mínimos cuadrados ordinarios (MCO); en los cinco (5) modelos de medición de la magnitud de los ajustes por devengo discrecionales $\left|\mathrm{DACC}_{\mathrm{it}}\right|$ y su interacción con las variables POST y de control, la R2 ajustada indica que los modelos número 2 (modelo de Jones Ajustado) y número 4 (modelo de Jones modificado con el ratio valor en libros sobre valor de mercado del patrimonio y cash flow operativo) son aquellos que presentan un mayor nivel de asociación entre dichas variables y su respectiva magnitud de ajustes discrecionales $(0.0929$ y 0.1287 respectivamente); adicionalmente, se evidencia que en los 5 modelos analizados, la relación entre los ajustes por devengo discrecionales y la variable POST resulta ser estadísticamente negativa en un nivel de significancia del 1\%, lo cual sugiere que las empresas utilizan los ajustes por devengo discrecionales en menor medida durante el periodo de aplicación de IFRS respecto a su periodo de pre-adopción, y esto es evidencia del efecto negativo que tiene las IFRS en el uso de dichos ajustes discrecionales (es consistente con la hipótesis número 2).

Respecto a las variables de control y la magnitud absoluta de los ajustes por devengo discrecionales, se observa una relación estadísticamente negativa entre las cinco expresiones utilizadas para medir dichos ajustes y las variables tamaño de las firmas (SIZE) y la cotización de las acciones de las compañías en un mercado de valores de los Estados unidos (XLIST), siendo no significativa la relación respecto a esta última variable; estos resultados sugieren que a mayor tamaño, las compañías latinoamericanas y del Caribe disminuyen el uso de los ajustes 
por devengo discrecionales o lo que es lo mismo, disminuye la manipulación del resultado, esto quizá se deba a que las más grandes compañías, sean seguidas en cuanto a su desempeño, por un mayor número de inversores y analistas.

Al tiempo, se observa una relación estadísticamente positiva entre la magnitud de los ajustes por devengo discrecionales bajo sus cinco expresiones y el nivel de endeudamiento ( $L E V)$, el cambio porcentual en las ventas (GROWHT), la gestión de activos (TURN) y los flujos de efectivo generados en la operación ( $C F O)$, lo cual sugiere incentivos para un mayor uso de los ajustes por devengo discrecionales, traduciéndose en mayor manipulación del resultado; esto posiblemente sugiere, para el caso del nivel de endeudamiento, que las compañías más endeudadas requieren el uso de los ajustes por devengo discrecionales para el cumplimiento de sus covenants. Se observa al igual una relación positiva entre los ajustes por devengo discrecionales y las variables prestación de los servicios de auditoría por medio de una firma Big4 (AUD), número de bolsas en que cotiza la acción de las compañías (NUMEX), y el reporte de pérdidas (LOSS), esto indica los incentivos o presiones que tienen las firmas latinoamericanas y del Caribe para aumentar el uso de la discrecionalidad en sus estimaciones contables.

Sin embargo, los resultados obtenidos son en gran medida, diferentes a los documentados por Chen et al. (2010), aplicados a países pertenecientes a la Unión Europea, en los que demuestra una relación negativa entre las distintas magnitudes de los ajustes por devengo discrecionales y los flujos de efectivo operativos, los servicios de auditoría prestados por una Big4, y el número de bolsas en las que cotice la acción de las compañías; esto indica que el entorno institucional en el que opera cada compañía, las débiles leyes de protección al inversor, la inexistencia del riesgo de litigio en varias economías y el grado de efectividad y desarrollo en los mecanismos de refuerzo o Enforcement (para garantizar la correcta aplicación de las normas contables), sugieren incentivos distintos que impulsan a cada una de ellas para manipular la información contable.

Table 6

Results of the regression of the absolute magnitude of discretionary accruals between the IFRS and the control variables.

\begin{tabular}{|c|c|c|c|c|c|c|}
\hline \multicolumn{7}{|c|}{ Dependent variable (5 models) } \\
\hline $\begin{array}{c}\text { Explicative } \\
\text { variable }\end{array}$ & $\begin{array}{l}\text { Expected } \\
\text { sign }\end{array}$ & $\left|D A C C_{i t}\right| \mathbf{1}$ & $\left|D A C C_{i t}\right| 2$ & $\left|D A C C_{i t}\right| 3$ & $\left|D A C C_{i t}\right| 4$ & $\left|D_{A C C} C_{i t}\right| \mathbf{5}$ \\
\hline Intercept & $?$ & $\begin{array}{l}0.082^{*} \\
(0.006)\end{array}$ & $\begin{array}{l}0.083 * \\
(0.006)\end{array}$ & $\begin{array}{l}0.083 * \\
(0.006)\end{array}$ & $\begin{array}{l}0.064 * \\
(0.006)\end{array}$ & $\begin{array}{c}0.08 * \\
(0.007)\end{array}$ \\
\hline POST & - & $\begin{array}{l}-0.013 * \\
(0.002)\end{array}$ & $\begin{array}{c}-0.013 * \\
(0.002)\end{array}$ & $\begin{array}{c}-0.013^{*} \\
(0.002)\end{array}$ & $\begin{array}{l}-0.008 * \\
(0.002)\end{array}$ & $\begin{array}{l}-0.012 * \\
(0.002)\end{array}$ \\
\hline SIZE & - & $\begin{array}{c}-0.003 * \\
(0.000)\end{array}$ & $\begin{array}{l}-0.003 * \\
(0.000)\end{array}$ & $\begin{array}{c}-0.003 * \\
(0.000)\end{array}$ & $\begin{array}{c}-0.003 * \\
(0.000)\end{array}$ & $\begin{array}{c}-0.003 * \\
(0.000)\end{array}$ \\
\hline LEV & + & $\begin{array}{l}0.004^{*} \\
(0.001)\end{array}$ & $\begin{array}{l}0.004 * \\
(0.001)\end{array}$ & $\begin{array}{l}0.004 * \\
(0.001)\end{array}$ & $\begin{array}{c}0.000 \\
(0.001)\end{array}$ & $\begin{array}{l}0.005 * \\
(0.001)\end{array}$ \\
\hline GROWTH & + & $\begin{array}{l}0.015^{*} \\
(0.003)\end{array}$ & $\begin{array}{l}0.015 * \\
(0.003)\end{array}$ & $\begin{array}{l}0.014 * \\
(0.003)\end{array}$ & $\begin{array}{l}0.007 * \\
(0.003)\end{array}$ & $\begin{array}{l}0.013 * \\
(0.003)\end{array}$ \\
\hline TURN & + & $\begin{array}{l}0.009 * \\
(0.002)\end{array}$ & $\begin{array}{l}0.009 * \\
(0.002)\end{array}$ & $\begin{array}{l}0.009 * \\
(0.002)\end{array}$ & $\begin{array}{l}0.013^{*} \\
(0.001)\end{array}$ & $\begin{array}{l}0.008 * \\
(0.002)\end{array}$ \\
\hline $\mathrm{CFO}$ & - & $\begin{array}{l}0.039 * \\
(0.010)\end{array}$ & $\begin{array}{l}0.036^{*} \\
(0.010)\end{array}$ & $\begin{array}{l}0.038 * \\
(0.010)\end{array}$ & $\begin{array}{l}0.027 * \\
(0.007)\end{array}$ & $\begin{array}{c}0.039 * \\
(0.01)\end{array}$ \\
\hline AUD & - & $\begin{array}{c}0.006 * * \\
(0.002)\end{array}$ & $\begin{array}{c}0.005 * * \\
(0.002)\end{array}$ & $\begin{array}{c}0.006 * * \\
(0.002)\end{array}$ & $\begin{array}{c}0.005 * * \\
(0.002)\end{array}$ & $\begin{array}{c}0.005 * * \\
(0.002)\end{array}$ \\
\hline NUMEX & - & $\begin{array}{c}0.000 \\
(0.001)\end{array}$ & $\begin{array}{c}0.000 \\
(0.001)\end{array}$ & $\begin{array}{c}0.000 \\
(0.001)\end{array}$ & $\begin{array}{c}0.002 * * \\
(0.001)\end{array}$ & $\begin{array}{l}-0.001 \\
(0.001)\end{array}$ \\
\hline
\end{tabular}


http://dx.doi.org/10.22201/fca.24488410e.2018.1233

\begin{tabular}{lccccc} 
LOSS & $0.025^{*}$ & $0.025^{*}$ & $0.025^{*}$ & $0.030^{*}$ & $0.023^{*}$ \\
& $(0.003)$ & $(0.003)$ & $(0.003)$ & $(0.002)$ & $(0.003)$ \\
XLIST & -0.002 & -0.002 & -0.002 & 0.000 & -0.001 \\
& $(0.004)$ & $(0.004)$ & $(0.004)$ & $(0.003)$ & $(0.004)$ \\
\hline R2 (Chi square) & 0.0984 & 0.0976 & 0.098 & 0.1346 & 0.0929 \\
Adjusted R2 (adjusted Chi & 0.0928 & 0.0929 & 0.0924 & 0.1287 & 0.0872 \\
square) & & & & & \\
F Value & 15.36 & 15.52 & 15.45 & 22.99 & 14.49 \\
Obs. & 5487 & 5487 & 5487 & 4020 & 5436 \\
\hline
\end{tabular}

Fuente: elaboración propia

$*, * *, * * *$ Indica diferencia significativa de cero con un nivel $\mathrm{p}<0.01, \mathrm{p}<0.05, \mathrm{p}<0.1$, respectivamente (prueba de dos colas). Se evalúa los supuestos del modelo de regresión Lineal bajo mínimos cuadrados ordinarios (MCO); no se evidencia multicolinealidad entre las regresoras, observándose los Factores de Inflación de la Varianza (VIF), que en su gran mayoría reportan valores menores a 5 . El test de White ejecutado a través del software STATA indica una probabilidad menor al 0.05 , lo que sugiere una alta probabilidad de falsedad en que la hipótesis de la varianza del término de error sean constantes, por tanto se asume el supuesto de homocedasticidad regresando nuevamente los modelos con el test Robusto de White en STATA. Se recurre al Teorema Central del Límite para asumir la normalidad en las perturbaciones de los modelos puesto que no es confirmado con el test de Shapiro-Wilk. El supuesto de autocorrelación es evaluado a través del test de Durbin-Watson y del test de Durbin, y para todos los modelos resulta una probabilidad mayor a 0.10 , lo cual indica poca probabilidad de falsedad en la hipótesis de no autocorrelación, por tanto se asume no autocorrelación entre los términos de error.

\section{Conclusiones}

Esta investigación tiene por objetivo comprobar si la calidad del reporte financiero (expresión de medición de la calidad contable) mejora después de la aplicación de las IFRS, para las compañías domiciliadas y cotizadas en los mercados de capitales latinoamericanos y del Caribe, que evidencian un cambio en su marco normativo contable desde sus GAAP locales a IFRS durante los periodos 2006 a 2014. Específicamente se examina si la aplicación de las IFRS en 15 países latinoamericanos y 10 países del Caribe, es asociada con menor magnitud de los ajustes por devengo discrecionales; dichos ajustes por devengo son obtenidos a través de cinco modelos, todos ellos partiendo del modelo básico de Jones (1991) y sus sucesivas modificaciones.

Después de controlar los efectos fijos de la industria y el país, que afectan la magnitud de los ajustes por devengo reportados por las compañías, los resultados obtenidos indican una disminución en el uso de los ajustes por devengo discrecionales (anormales), visto desde una menor magnitud absoluta en dichos ajustes durante los periodos de aplicación de IFRS, y desde una relación estadísticamente negativa entre estas dos últimas variables; por lo tanto, esto es indicativo de un menor nivel de manipulación del resultado (earnings management), lo que es lo mismo, un mejoramiento significativo en la calidad del reporte financiero (calidad contable). Los resultados obtenidos en este estudio multi-país latinoamericano, corresponden con lo documentado sobre varias economías latinas consideradas individualmente, por ejemplo con lo documentado por Pelucio-Grecco et al. (2014) para el caso brasileño y por Conesa et al. (2011) para el caso mexicano.

Respecto a los incentivos de la gerencia que impulsan hacia un mayor uso de los ajustes por devengo discrecionales, se pudo constatar, a través de los resultados, que el entorno institucional en el que opera cada empresa junto con el nivel de desarrollo de leyes de protección al inversor y la efectividad de los mecanismos de refuerzo (Enforcement) para garantizar la calidad del reporte financiero, condicionan que dichos incentivos difieran de un país a otro; siendo contrario a lo documentado para la Unión Europea (Chen et al., 2010) y sorprendente en alguna medida, 
el hecho que una compañía latinoamericana o Caribeña obtenga flujos de efectivos operativos positivos, cotice en varios mercados de capitales o sea auditada por una firma perteneciente a las Big4, y esto determina el uso de un mayor nivel de manipulación del resultado (incremento en la magnitud absoluta de los ajustes por devengo discrecionales).

Partiendo de la limitación identificada en la literatura previa, en la que los hallazgos sobre la calidad contable no podrían generalizarse a nivel latinoamericano y del Caribe, puesto que sólo se disponía de información para un país en particular durante un rango limitado de años de aplicación de IFRS, este articulo contribuye a la literatura resolviendo esta limitación al presentar los resultados generalizados para la región latina y del Caribe, incluyendo en el estudio, a casi la totalidad de los países de esta región durante un amplio periodo de análisis de IFRS (2006-2014), además de utilizar varias métricas de medición para dar mayor robustez a los hallazgos sobre uso de estos estándares internacionales en la calidad del reporte financiero en dichos países, considerados como aquellos con influencia francesa en la definición de su sistema contable (La Porta, Lopez De Silanes, Shleifer y Vishny, 1997).

Futuras investigaciones podrían corroborar el nivel de manipulación del resultado para los países latinoamericanos y del Caribe entre sí, incluso identificar las diferencias en el grado de discrecionalidad de los ajustes por devengo entre las principales economías latinoamericanas respecto a países con sistema contable de influencia anglosajona, escandinava o germánica (La Porta et al., 1997).

\section{References}

Adibah Wan Ismail, W., Anuar Kamarudin, K., van Zijl, T., \& Dunstan, K. (2013). Earnings quality and the adoption of IFRS-based accounting standards: Evidence from an emerging market. Asian Review of Accounting, 21(1), 53-73. http://dx.doi.org/10.1108/13217341311316940

Ahmed, A. S., Neel, M., \& Wang, D. (2013). Does mandatory adoption of IFRS improve accounting quality? Preliminary evidence. Contemporary Accounting Research, 30(4), 1344-1372. http://dx.doi.org/10.1111/j.19113846.2012.01193.x

Aussenegg, W., Inwinkl, P., \& Schneider, G. T. (2008). Earnings management and local vs. international accounting standards of European public firms. https://dx.doi.org/10.2139/ssrn.1310346

Brad, L., Dobre, F., \& Ciobanu, R. (2014). Evidence of no difference between the values of discretional accruals computed using the Romanian accounting standards and the international financial reporting standards. Amis 2014, n.d., 556-567. http://dx.doi.org/10.13140/2.1.4756.2247

Bryce, M., Ali, M. J., \& Mather, P. R. (2015). Accounting quality in the pre-/post-IFRS adoption periods and the impact on audit committee effectiveness-Evidence from Australia. Pacific-Basin Finance Journal, 35, $163-181$. https://doi.org/10.1016/j.pacfin.2014.12.002

Callao, S., \& Jarne, J. I. (2010). Have IFRS affected earnings management in the European Union? Accounting in Europe, 7(2), 159-189. http://dx.doi.org/10.1080/17449480.2010.511896

Chen, H., Tang, Q., Jiang, Y. \& Lin, Z. (2010). The role of international financial reporting standards in accounting quality: Evidence from the European Union. Journal of International Financial Management \& Accounting, 21(3), 220-278. http://dx.doi.org/10.1111/j.1467-646X.2010.01041.x

Conesa, I. M., Manzano, M. P., \& Sánchez, H. G. (2011). La calidad del resultado pre y post-adaptación a las IFRS en México por el CINIF. (Disponible en: http://www.aeca1.org/pub/on_line/comunicaciones_aal2011/cd/54a.pdf) y (Consultado: 15/05/2017) 
DeAngelo, L. E. (1986). Accounting numbers as market valuation substitutes: A study of management buyouts of public stockholders. Accounting Review, n.d, 400-420. (Disponible en: https://www.jstor.org/stable/247149) y (Consultado: 15/05/2017)

Dechow, P. M., Richardson, S. A. \& Tuna, I. (2003). Why are earnings kinky? an examination of the earnings management explanation. Review of Accounting Studies, 8(2-3), 355-384. http://dx.doi.org/10.1023/A:1024481916719

Dechow, P. M., Sloan, R. G. \& Sweeney, A. P. (1995). Detecting earnings management. Accounting Review, 70(2), 193-225. (Disponible en: http://www.jstor.org/stable/248303) y (Consultado: 15/05/2017)

Defond, M. L. \& Jiambalvo, J. (1994). Debt covenant violation and manipulation of accruals. Journal of Accounting \& Economics, 17(1-2), 145-176. http://dx.doi.org/10.1016/0165-4101(94)90008-6

Dimitropoulos, P. E., Asteriou, D., Kousenidis, D., \& Leventis, S. (2013). The impact of IFRS on accounting quality: Evidence from Greece. Advances in Accounting, 29(1), 108-123. https://doi.org/10.1016/j.adiac.2013.03.004

Doukakis, L. C. (2014). The effect of mandatory IFRS adoption on real and accrual-based earnings management activities. Journal of Accounting and Public Policy, 33(6), 551-572. https://doi.org/10.1016/j.jaccpubpol.2014.08.006

Friedlan, J. M. (1994). Accounting choices of issuers of initial public offerings. Contemporary Accounting Research, 11(1), 1-31. http://dx.doi.org/10.1111/j.1911-3846.1994.tb00434.x

Gray, S. J., Kang, T., Lin, Z., \& Tang, Q. (2015). Earnings management in Europe post IFRS: Do cultural influences persist? Management International Review, 55(6), 827-856. http://dx.doi.org/10.1007/s11575-015-0254-7

Healy, P. M. (1985). The effect of bonus schemes on accounting decisions. Journal of Accounting and Economics, 7(1-3), 85-107. https://doi.org/10.1016/0165-4101(85)90029-1

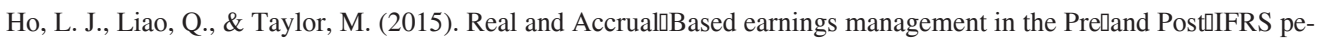
riods: Evidence from china. Journal of International Financial Management \& Accounting, 26(3), 294-335. http:// dx.doi.org/10.1111/jifm.12030

Houqe, M. N., van Zijl, T., Dunstan, K., \& Karim, A. W. (2012). The effect of IFRS adoption and investor protection on earnings quality around the world. The International Journal of Accounting, 47(3), 333-355. https://doi. org/10.1016/j.intacc.2012.07.003

Iatridis, G. (2010). International financial reporting standards and the quality of financial statement information. International Review of Financial Analysis, 19(3), 193-204. https://doi.org/10.1016/j.irfa.2010.02.004

Istrate, C., Eugenia, I., Carp, M., Bogdan Robu, I., \& Pavaloaia, L. (2015). Accruals earnings management in emerging markets under the transition to IFRS: The case of Romanian listed companies. Transformations in Business \& Economics, 14(2 A), 393-411. (Disponible en: https://www.researchgate.net/profile/Ioan-Bogdan_Robu/publication/290820080_Accruals_earnings_management_in_emerging_markets_under_the_transition_to_IFRS_The_ case_of_Romanian_listed_companies/links/569bfd7708aea147695466b8.pdf) y (Consultado: 15/05/2017)

Jones, J. J. (1991). Earnings management during import relief investigations. Journal of Accounting Research, 29(2), 193-228. http://dx.doi.org/10.2307/2491047

Jong-Il, P. (2015). The Effect of IFRS Adoption on Real Earnings Management. 세무와 회계저널, 16(5), 65-110. (Disponible en: http://www.papersearch.net/thesis/article.asp?KEY=3369225) y (Consultado: 15/05/2017)

Kim, J. (2013). The effect of adopting K-IFRS on financial Reports-Case study with KT\&G. International Journal of Multimedia and Ubiquitous Engineering. n.d, 1-8. (Disponible en http://www.sersc.org/journals/IJMUE/ vol8_no2_2013/19.pdf) y (Consultado: 15/05/2017)

Kim, K. (2014). The association between K-IFRS adoption and earnings management: Focusing on external auditor size. Accounting, Tax \& Auditing Research, 56(1), 117-146. (Disponible en: https://www.google.es/ url?sa=t\&rct=j\&q=\&esrc=s\&source=web\&cd=1\&cad=rja\&uact=8\&ved=0ahUKEwjU1dbz1a_UAhXMnBoKHbt3BQAQFggpMAA\&url=http\%3A\%2F\%2Fcentral.oak.go.kr\%2Fjournallist\%2Farticlepdf.do\%3Bjsessionid\%3DAC7AB273A01DD37715F2F9C8A91F8750\%3Fur1\%3D\%2Frepository\%2Fjournal\%2F19105\%2FNRF027_2014_v56n1_117.pdf\&usg=AFQjCNG8pAYQ_KLM1RjWNge4JkB-1GolyQ) y (Consultado: 15/05/2017)

Kothari, S. P., Leone, A. J. \& Wasley, C. E. (2005). Performance matched discretionary accrual measures. Journal of Accounting \& Economics, 39(1), 163-197. http://dx.doi.org/10.1016/j.jacceco.2004.11.002

Kwon, S., \& Whan Oh, J. (2015). Attributes of K-IFRS Reported Earnings. 대한경영학회지, 28(3), 1047-1064. (Disponible en: http://www.dbpia.co.kr/Journal/ArticleDetail/NODE06270524) y (Consultado: 15/05/2017) 
La Porta, R., Lopez De Silanes, F., Shleifer, A. \& Vishny, R. M. (1997). Legal determinants of external finance. Journal of Finance, 52(3), 1131-1150. http://dx.doi.org/10.1111/j.1540-6261.1997.tb02727.x

Larcker, D. F. \& Richardson, S. A. (2004). Fees paid to audit firms, accrual choices, and corporate governance. Journal of Accounting Research, 42(3), 625-658. http://dx.doi.org/10.1111/j.1475-679X.2004.t01-1-00143.x

Leung, R. (2015). Earnings management motives and firm value following mandatory IFRS Adoption-Evidence from Canadian companies. http://dx.doi.org/10.2139/ssrn.2688220

Liu, C., Yip Yuen, C., J. Yao, L., \& H. Chan, S. (2014). Differences in earnings management between firms using US GAAP and IAS/IFRS. Review of Accounting and Finance, 13(2), 134-155. https://doi.org/10.1108/RAF-10-20120098

Osma, B. G., Enguídanos, A. M., Clemente, A. G., \& Lara, J. M. G. (2004). La comparabilidad de la información contable en Europa: efectos de la manipulación contable sobre el nivel de conservadurismo. Trabajo presentado en Congreso AECA. Universidad de Valencia. (Disponible en: http://www.ivie.es/downloads/docs/wpasec/wpasec-2004-14.pdf) y (Consultado: 15/05/2017)

Palacios Manzano, M., \& Martinez Conesa, I. (2014). Assessing the impact of IFRS adaptation on earnings management: An emerging market perspective. Transformation in Business \& Economics, 13(1), 21-40. (Disponible en: http://www.transformations.knf.vu.lt/31/ge31.pdf) y (Consultado: 15/05/2017)

Pelucio-Grecco, M. C., Geron, C. M. S., Grecco, G. B., \& Lima, J. P. C. (2014). The effect of IFRS on earnings management in Brazilian non-financial public companies. Emerging Markets Review, 21, 42-66. https://doi.org/10.1016/j.ememar.2014.07.001

Rudra, T., \& Bhattacharjee, C. D. (2012). Does IFRs influence earnings management? Evidence from India. Journal of Management Research, 4(1), 1-13. https://doi.org/10.5296/jmr.v4i1.849

Salewski, M., Teuteberg, T., \& Zülch, H. (2014). Short-term and long-term effects of IFRS adoption on disclosure quality and earnings management. http://dx.doi.org/10.2139/ssrn.2398305

Sang-Ae, P., Nam-Ryoung, L., \& Sang-Bum, P. (2011). Earnings Quality Changes Around IFRS Adoption: Evidence from an Emerging Market. 대한경영학회지, 24(1), 73-96. (Disponible en: http://www.dbpia.co.kr/Journal/ArticleDetail/NODE01639733) y (Consultado: 15/05/2017)

Santana, V., Timm, A., Costa, I., \& Zoboli, F. (2014). IFRS accounting quality in Latin America: A comparison with Anglo-Saxon and continental European countries and the role of cross listing in the US. (Disponible en: http:// papers.ssrn.com/sol3/papers.cfm?abstract_id=2438268) y (Consultado: 15/05/2017)

Van Tendeloo, B. \& Vanstraelen, A. (2005). Earnings management under German GAAP versus IFRS. European Accounting Review, 14(1), 155-180. http://dx.doi.org/10.1080/0963818042000338988

Watrin, C., \& Ullmann, R. (2012). Improving earnings quality: The effect of reporting incentives and accounting standards. Advances in Accounting, 28(1), 179-188. https://doi.org/10.1016/j.adiac.2012.03.001

Zeghal, D., Chtourou, S. M., \& Fourati, Y. M. (2012). The effect of mandatory adoption of IFRS on earnings quality: Evidence from the European Union. Journal of International Accounting Research, 11(2), 1-25. https://doi. org/10.2308/jiar-10221

Zéghal, D., Chtourou, S., \& Sellami, Y. M. (2011). An analysis of the effect of mandatory adoption of IAS/IFRS on earnings management. Journal of International Accounting, Auditing and Taxation, 20(2), 61-72. https://doi. org/10.1016/j.intaccaudtax.2011.06.001

Zhang, Y., Uchida, K., \& Bu, H. (2013). How do accounting standards and insiders' incentives affect earnings management? Evidence from china. Emerging Markets Review, 16, 78-99. https://doi.org/10.1016/j.ememar.2013.04.002 